\title{
Effects of Acoustic Heterogeneity in Breast Thermoacoustic Tomography
}

\author{
Yuan Xu and Lihong V. Wang, Senior Member, IEEE
}

\begin{abstract}
The effects of wavefront distortions induced by acoustic heterogeneities in breast thermoacoustic tomography (TAT) are studied. Amplitude distortions are shown to be insignificant for different scales of acoustic heterogeneities. For wavelength-scale, or smaller, heterogeneities, amplitude distortion of the wavefront is minor as a result of diffraction when the detectors are placed in the far field of the heterogeneities. For larger-scale heterogeneities at the parenchyma wall, by using a ray approach (geometric optics), we show that no refraction-induced multipath interference occurs and, consequently, that no severe amplitude distortion, such as is found in ultrasound tomography, exists. Next, we consider the effects of phase distortions (errors in time-of-flight) in our numerical studies. The numerical results on the spreads of point sources and boundaries caused by the phase distortions are in good agreement with the proposed formula. After that, we demonstrate that the blurring of images can be compensated for by using the distribution of acoustic velocity in the tissues in the reconstructions. The effects of the errors in the acoustical velocities on this compensation also are investigated. An approach to implement the compensation using only TAT data is proposed. Lastly, the differences in the effects of acoustic heterogeneity and the generation of speckles in breast TAT and breast ultrasound imaging are discussed.
\end{abstract}

\section{INTRODUCTION}

$\mathrm{W}$ HEN an electromagnetic pulse is absorbed by biological tissue, the heating and subsequent expansion causes the emission of acoustic signals; this phenomenon is called the thermoacoustic effect. In thermoacoustic tomography (TAT), the thermoacoustic signals from a tissue sample are collected to map the distribution of the radiation absorption within the sample. Radiation absorption is closely related to the physiological and pathological status of the tissue. For example, the microwave absorption rate of cancerous breast tissue is two to five times greater than that of the surrounding normal breast tissue. This difference has been attributed to an increase in the amount of bound water and sodium within malignant cells [1]-[3].

The TAT combines good imaging resolution with high imaging contrast. Microwave imaging alone has the ad-

Manuscript received March 8, 2002; accepted February 25, 2003. This project was sponsored in part by the U.S. Army Medical Research and Material Command Grant No. DAMD17-00-1-0455, the National Institutes of Health Grants No. R01 CA71980 and No. R21 CA83760, the National Science Foundation Grant No. BES-9734491, and Texas Higher Education Coordinating Board Grant No. ARP 000512-0063-2001.

The authors are with the Optical Imaging Laboratory, Department of Biomedical Engineering, Texas A\&M University, College Station, TX 77843-3120 (e-mail: lwang@tamu.edu). vantage of good imaging contrast but suffers from poor spatial resolution [4]-[7]. On the other hand, purely ultrasonic imaging has good spatial resolution but poor contrast. TAT capitalizes on the advantages of both methods.

There are a variety of reconstruction algorithms for TAT [8]-[13]. By using the approximation that the distance between the detector and the absorbing object is much larger than the dimensions of the absorbing object, a three-dimensional (3-D) radon transform has been used to reconstruct objects in TAT [8]. A time-domain, focusedbeam-forming technique also has been applied to image reconstruction in the photoacoustic scanning of tissue structures [9], and a delay-and-sum algorithm has been applied to microwave-induced TAT [12]. The above reconstructions were implemented in the time domain. In the frequency domain, exact reconstruction algorithms for TAT have been implemented in planar, cylindrical, and spherical configurations with series expansion techniques [11]-[13].

An important assumption in the above reconstruction algorithms is that the tissue is acoustically homogeneous. For many medical imaging applications, including imaging of the female breast, this assumption is an approximation. For example, the speed of sound in the breast can vary from $1400 \mathrm{~m} / \mathrm{s}$ to $1550 \mathrm{~m} / \mathrm{s}$. Errors due to the assumption of a constant acoustic speed, which has never been studied in TAT, potentially can have a pronounced effect on image quality. In breast ultrasound tomography (UT), however, wavefront distortion has been studied extensively [14]-[17]. The amplitude distortion caused by refraction dominates the phase distortion induced by acoustic speed variation in the breast UT [15]. Refraction occurs where there is a speed mismatch across a tissue interface. Because of refraction, rays from a single source can reach the same receiver by different paths, as shown in Fig. 1. The interference between these rays causes strong amplitude distortions in breast UT. Different deaberration methods have been proposed to compensate for phase distortion in UT [18], [19]. However, so far they have been inadequate to correct the strong amplitude distortion caused by refraction [20].

The effects of acoustic heterogeneity on breast TAT are estimated to be weaker than those in breast UT for the following reasons. First, signals in breast TAT are primarily in a lower frequency range (usually below $1.5 \mathrm{MHz}$ [21]) than those in UT. Ultrasound scattering in this frequency range is weak. Second, in TAT, the acoustic source is induced by electromagnetic absorption; therefore, only oneway distortion on reception wave propagation occurs. As shown in Fig. 2, an acoustic ray, for example $S B_{1} D$, needs to pass through interface $\Sigma$ only once. In contrast, in pure 


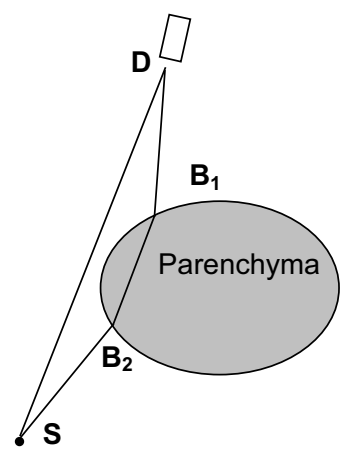

Fig. 1. Multipath interference caused by refraction at boundary points $B_{1}$ and $B_{2}$ in breast ultrasound imaging in transmission mode. $S$ is a point source and $D$ is a detector.

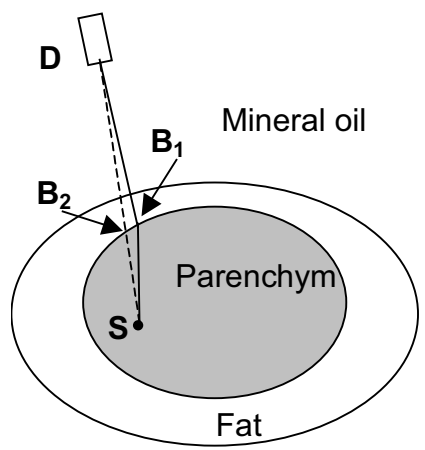

Fig. 2. Ray refraction at the parenchyma wall with breast TAT. The outer oval represents the breast surface, in which there is negligible refraction due to the good match of acoustic speed between fat and mineral oil. The solid line $S B_{1} D$ represents a ray in the heterogeneous model; the dashed line $S B_{2} D$ represents that in a homogeneous model. $S$ is a point source and $D$ is a detector; $B_{1}, B_{2}$ are two points at the parenchyma wall.

ultrasound imaging, either in the pulse-echo mode or in the transmission mode, ultrasound distortion includes two parts: distortion during both transmission and reception wave propagation. Therefore, the acoustic wave has to pass through the interface at least twice, as shown in $S B_{2} B_{1} D$ in Fig. 1. Third, if the detection distance from the objects are properly chosen, the effects of amplitude distortion can be minimized in breast TAT, as will be shown in Section III.

In our work, we analyze the effects of amplitude distortion and numerically simulate the effects of phase distortion with the truncated conjugate gradient [22] (TCG) method. In Section II, we derive equations for the forward problem in an acoustically homogeneous model, which yields acoustic pressure from a known distribution of microwave absorption. In Section III, we investigate the effects of refraction on the wavefront amplitude and phase in breast TAT. We prove that, in breast TAT, a convex parenchyma wall (when observed from the outside of the parenchyma tissue) does not cause multipath interference and that the effects of amplitude distortion also are not severe for a concave boundary. An equation for the for- ward problem in an acoustically heterogeneous model also is introduced at the end of Section III. The inversion algorithm of TCG, and the model and parameters used in the numerical simulations, are presented in Section IV. In Section V, the effects of phase distortion are studied numerically. We show how the degradation of the reconstructed images depends on acoustic heterogeneity when acoustic heterogeneity is not considered in the reconstruction algorithm. Correction of phase distortion should be the first step for improving image quality in breast TAT because phase is much more important in imaging than amplitude when there is no severe amplitude distortion [23], [24]. Therefore, the reconstructions are implemented with consideration of acoustic velocity heterogeneity to illustrate how the imaging degradation can be compensated for. The effects of the errors in the acoustical velocities on this compensation also are investigated. In Section VI, an approach to implement compensation with only TAT data is proposed. The differences between breast TAT and breast ultrasound imaging on the effects of acoustic heterogeneity and speckles are explained by their differences in central ultrasound frequency and detection geometry. Section VII presents conclusions.

\section{The Forward Problem in a Homogeneous MODEL}

We begin by deriving a formula for the forward problem for an acoustically homogeneous model, then modify it at the end of Section III to consider velocity heterogeneity. In the case of thermal confinement, the acoustic wave at point $\mathbf{r}$ and time $t, p(\mathbf{r}, t)$, is related to the microwave absorption $H(\mathbf{r}, t)$ by the following wave equation [25]:

$$
\frac{\partial^{2} p(\mathbf{r}, t)}{\partial t^{2}}-v_{s 0}^{2} \nabla^{2} p(\mathbf{r}, t)=\frac{\beta}{C} \frac{\partial H(\mathbf{r}, t)}{\partial t},
$$

where $v_{s 0}$ is the acoustic speed, $C$ is the specific heat, and $\beta$ is the coefficient of the volume thermal expansion. (1) can be rewritten in terms of $H(\mathbf{r}, t)$ :

$$
p(\mathbf{r}, t)=\frac{\beta}{4 \pi C} \iiint \frac{\partial H\left(\mathbf{r}^{\prime}, t^{\prime}\right)}{\partial t^{\prime}} \frac{d \mathbf{r}^{\prime}}{\left|\mathbf{r}-\mathbf{r}^{\prime}\right|},
$$

where $t^{\prime}=t-\left|\mathbf{r}-\mathbf{r}^{\prime}\right| / v_{s}$. The source term $H(\mathbf{r}, t)$ can further be written as the product of a spatial component and a temporal component, i.e.:

$$
H(\mathbf{r}, t)=I_{0} \varphi(\mathbf{r}) \eta(t)
$$

where $I_{0}$ is a scaling factor proportional to the incident radiation intensity, $\varphi\left(\mathbf{r}^{\prime}\right)$ describes the to-be-reconstructed microwave absorption properties of the medium at $\mathbf{r}^{\prime}$, and $\eta(t)$ describes the shape of the irradiating pulse. Substituting (3) into (4) results in:

$$
p(\mathbf{r}, t)=\frac{\beta I_{0}}{4 \pi C} \iiint \varphi\left(\mathbf{r}^{\prime}\right) \frac{d \eta\left(t^{\prime}\right)}{d t^{\prime}} \frac{d \mathbf{r}^{\prime}}{\left|\mathbf{r}-\mathbf{r}^{\prime}\right|} .
$$


We proceed by transforming the time-dependent wave equation into the temporal-frequency domain. Denoting the Fourier transforms of $p$ and $\eta$ by $\bar{p}$ and $\bar{\eta}$, respectively, we have:

$$
\begin{aligned}
p(\mathbf{r}, t) & =\int_{-\infty}^{\infty} \bar{p}(\mathbf{r}, k) \exp (i k t) d k, \\
\eta(t) & =\int_{-\infty}^{\infty} \bar{\eta}(k) \exp (i k t) d k .
\end{aligned}
$$

Substituting (5) into (4) results in:

$$
\bar{p}(\mathbf{r}, k)=\frac{i \beta I_{0} k \bar{\eta}(k)}{4 \pi C} \iiint \varphi\left(\mathbf{r}^{\prime}\right) \frac{\exp \left(-i k\left|\mathbf{r}-\mathbf{r}^{\prime}\right| / v_{s 0}\right)}{\left|\mathbf{r}-\mathbf{r}^{\prime}\right|} d \mathbf{r}^{\prime}
$$

Define $\bar{p}_{1}(\mathbf{r}, k)=\bar{p}(\mathbf{r}, k) /(2 \pi \eta(k))$, substitute it into (6), apply an inverse Fourier transform to both sides of the equation, and obtain the following equation:

$$
p_{1}(\mathbf{r}, t)=\frac{v_{s 0} \beta I_{0}}{4 \pi C} \frac{\partial}{\partial t} \iint_{t=t_{f}\left(\mathbf{r}^{\prime}, \mathbf{r}\right)} \frac{\varphi\left(\mathbf{r}^{\prime}\right)}{\left|\mathbf{r}-\mathbf{r}^{\prime}\right|} d \mathbf{r}^{\prime},
$$

where

$$
t_{f}\left(\mathbf{r}^{\prime}, \mathbf{r}\right)=\left|\mathbf{r}-\mathbf{r}^{\prime}\right| / v_{s 0}
$$

is the time-of-flight (TOF) from to $\mathbf{r}^{\prime}$ to $\mathbf{r} ; p_{1}(\mathbf{r}, t)$ is the deconvolution of $p(\mathbf{r}, t)$ with respect to the length of the microwave pulse and can be interpreted as the detected pressure signal when the microwave pulse is infinitely narrow. The physical meaning of this equation is that, in an acoustically homogenous medium, the pressure $p_{1}$, at a spatial point $\mathbf{r}$ and time $t$, is proportional to the first-order temporal derivative of the integration of the absorbed microwave energy over a spherical surface [a circle in the twodimensional (2-D) case]. The spherical surface is centered at $\mathbf{r}$ and has a radius of $t v_{s 0}$.

\section{The Effect of Acoustic Heterogeneity IN TAT}

A TAT model is shown in Fig. 2. In our imaging system, mineral oil is chosen as the coupling medium for both microwaves and ultrasonic waves. The acoustic speed in mineral oil is $1437 \mathrm{~m} / \mathrm{s}$ [26], which is very close to that in fat [27]. Therefore, there should be negligible refraction at the boundary between the breast and the mineral oil; consequently, we will consider only the effects of the acoustical heterogeneity within the breast. More details on our TAT experimental setup can be found in [12].

\section{A. Amplitude Distortion Caused by Refraction}

Fig. 1 shows the multipath interference in breast ultrasound imaging in transmission mode. The acoustic ray from source $S$ can travel to detector $D$ by two different paths, $S D$ and $S B_{2} B_{1} D$, due to refraction at the interfaces between different tissues. The interference between

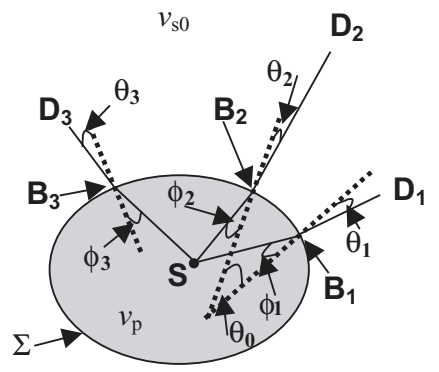

Fig. 3. Diagram showing that no two rays from a point source $S$ will intersect with each other after being refracted at a convex boundary $\Sigma$ and entering a medium with a slower acoustic speed. $S$ is a point source; $D_{1}$ and $D_{2}$ are detectors; $\phi_{1}, \phi_{2}$, and $\phi_{3}$ are the incidence angles; $\theta_{1}, \theta_{2}$, and $\theta_{3}$ are the transmission angles; the solid lines represent acoustic rays; $B_{1}, B_{2}, B_{3}$ are three points at the parenchyma wall; $v_{p}, v_{s 0}$ are the mean acoustic speeds in the parenchyma tissue and the fat tissue, respectively; and $v_{p}>v_{s 0}$.

the two rays can cause amplitude distortion [15]. In the following subsections, we will first prove that there is no multipath interference in the case of a convex parenchyma wall in breast TAT. Then, we will show that the amplitude distortion also is not severe for a concave parenchyma wall.

1. Convex Boundary: In this subsection, we will show that there is no multipath interference in the TAT of the breast with a convex parenchyma wall by proving that no two rays from a source within the parenchyma will intersect with each other after refractions at the wall. The model is shown in Fig. 3, where $S$ is an acoustic source; $v_{p}$ and $v_{s 0}$ are the acoustic speed in the breast parenchyma and the medium (also the fat), respectively $\left(v_{p}>v_{s 0}\right)$; the dashed lines are the normals of the boundary at points $B_{1}$, $B_{2}, B_{3}$, respectively; $\phi_{1}, \phi_{2}$, and $\phi_{3}$ are the angles of incidence; $\theta_{1}, \theta_{2}$, and $\theta_{3}$ and are the angles of transmission; and the solid lines represent the acoustic rays. Because the boundary is convex, it can be inferred that rotation from the normal at point $B_{2}$ to the normal at point $B_{1}$ is clockwise and the angle is $\theta_{0}$ (positive). We also have $\phi_{2}<\theta_{0}+\phi_{1}$, which can be seen by extending lines $S B_{2}$ and $S B_{1}$ to the outside of the boundary and noticing that $S B_{2}$ and $S B_{1}$ will never intersect outside the boundary. To prove $B_{2} D_{2}$ and $B_{1} D_{1}$ will not intersect outside the boundary, we need to show $\theta_{2}<\theta_{0}+\theta_{1}$. According to Snell's law, we have:

$$
\begin{aligned}
& \sin \theta_{2}=(1-\alpha) \sin \phi_{2}, \\
& \sin \theta_{1}=(1-\alpha) \sin \phi_{1},
\end{aligned}
$$

where $\alpha=1-v_{s 0} / v_{p}$, which is positive when $v_{p}>v_{s 0}$. The problem can be discussed under two conditions:

$\phi_{2}<\phi_{1}$. In this case, according to (9), we have $\theta_{2}<\theta_{1}$ and therefore $\theta_{2}<\theta_{0}+\theta_{1}$. And

$\phi_{2} \geq \phi_{1}$. (9) can be transformed to:

$$
\begin{aligned}
& \sin \left(\frac{\theta_{1}-\phi_{1}}{2}\right)=-\frac{\alpha \sin \left(\phi_{1}\right)}{2 \cos \left(\left(\theta_{1}+\phi_{1}\right) / 2\right)}, \\
& \sin \left(\frac{\theta_{2}-\phi_{2}}{2}\right)=-\frac{\alpha \sin \left(\phi_{2}\right)}{2 \cos \left(\left(\theta_{2}+\phi_{2}\right) / 2\right)} .
\end{aligned}
$$


Because $\phi_{2} \geq \phi_{1}$ and consequently $\theta_{2} \geq \theta_{1}$, it is straightforward to obtain $\theta_{2}-\phi_{2} \leq \theta_{1}-\phi_{1}$ from (10). Using $\phi_{2}<\theta_{0}+\phi_{1}$, we have $\theta_{2}<\theta_{0}+\theta_{1}$. In conclusion, we prove that, after the rays from a point source go into another medium with a slower acoustic speed, the rays cannot intersect with each other when the interface is convex. In another words, for any pairing of point source and detector, there is only one acoustic path that satisfies Snell's law. Consequently, no multipath interference occurs and amplitude distortion can be ignored. This conclusion also can be applied to a boundary with wavelength-scale concave segments. This kind of boundary can be treated as a convex boundary approximately because the effects of the small concave segments can be neglected when the detectors are placed in the far field of the segments, as will be shown in the following subsection. In contrast, multipath interference does occur after rays pass a convex parenchyma wall in ultrasound imaging, as shown in Fig. 1. This difference makes the amplitude distortion in TAT of the breast with a convex, or approximately convex parenchyma wall, smaller than that in pure ultrasound imaging.

2. Concave Boundary: We realize that, in reality, the boundary between mammary tissue and subcutaneous fat tissue might be concave and quite irregular. In this subsection, we will show that the amplitude distortion caused by a concave boundary is not severe. Basically, this conclusion can be explained as follows. With wavelength-scale or smaller heterogeneities, amplitude distortion of the wavefronts is minor due to diffraction when the detectors are placed in the far field of the irregular boundary segment. When the size of the concave segment is larger, according to the imaging formula of concave boundaries shown below, only imaginary images exist after the wavefronts from real objects pass through the concave boundary. Equivalently, no two rays from a point source will intersect with each other after passing through the concave boundary segment and no strong amplitude distortion occurs. In the following subsection, we will define two kinds of multipath interference: focusing-type and nonfocusing-type interferences. The former can induce amplitude distortion in both narrowband and broadband signals; the latter can induce only amplitude distortion in narrowband signals. As a consequence, we need only examine in detail the focusing-type interference, because signals in breast TAT are broadband.

Definition of focusing-type and nonfocusing-type interferences. Fig. 4 shows the two different kinds of multipath interferences. Three different ray paths $S B_{1} D, S B_{2} D$, $S B_{3} D$ from source $S$ to detector $D$ are shown, and each of them is assumed to satisfy the refraction law. The $S B_{1} D$ and $S B_{2} D$ can be considered as a small modification of the straight line $S D$ due to weak heterogeneity, and $S B_{3} D$ is far away from $S D$. We use focusing-type interference to refer to the interference between pulses along the paths with the same TOFs. The interference between $S B_{1} D$ and $S B_{2} D$ is of this type. This is because $S B_{1} D$ and $S B_{2} D$ satisfy the refraction law, and their TOFs are local minima according to Fermat's principle [28]. Conse-

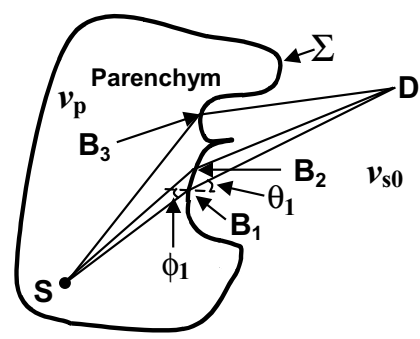

Fig. 4. Diagram to show two types of multipath interferences caused by a concave boundary: focusing-type interference between $S B_{1} D$ and $S B_{2} D$ and nonfocusing-type interference between $S B_{1} D$ $\left(S B_{2} D\right)$ and $S B_{3} D$. In a focusing-type interference, the different rays have approximately the same TOF, which consequently yields constructive interference and strong amplitude distortion. In this case, the boundary segment around $B_{1} B_{2}$ can be considered as a lens. In nonfocusing-type interference, the difference of the TOFs along two rays is larger than the pulse width; consequently, the pulses are separated temporally and no strong amplitude distortion occurs. See Fig. 3 for the symbols' definitions.

quently the rays around $S B_{1} D$ and $S B_{2} D$ should have almost the same TOF. After noticing that $B_{1}$ and $B_{2}$ are close to each other, it can be inferred that $S B_{1} D$ and $S B_{2} D$ have the same TOFs. Actually, the boundary segment around $B_{1} B_{2}$ can be considered a focusing lens and can produce strong amplitude distortion even for broadband pulses, as verified by the strong amplitude distortion in broadband breast ultrasound imaging [15]. As a contrast, we use nonfocusing-type interference to refer to the interference between the pulses along paths with different TOFs. The interference between $S B_{3} D$ and $S B_{1} D$ $\left(S B_{2} D\right)$ is a nonfocusing-type interference, because $B_{3}$ is far from $B_{1}$ and $B_{2}$, and generally it can be assumed that $\left|t_{S B_{1} D}-t_{S B_{3} D}\right|$ and $\left|t_{S B_{2} D}-t_{S B_{3} D}\right|\left(t_{S B_{1} D}, t_{S B_{2} D}\right.$, and $t_{S B_{3} D}$ are the TOFs along ray paths $S B_{1} D, S B_{2} D$, and $S B_{3} D$, respectively) are larger than $1 \mu \mathrm{s}$, the average pulse width of thermoacoustic signals in our RF TAT experiments. Consequently, the pulse along $S B_{3} D$ is separated temporally from the pulses along $S B_{1} D, S B_{2} D$, and the interference between $S B_{3} D$ and $S B_{1} D\left(S B_{2} D\right)$ is insignificant. Similar analyses can be found in the pure ultrasound imaging literature [15].

The signals along $S B_{3} D$ may introduce artifacts in the reconstructed images because detector $D$ receives two pulses from source $S$-one along $S B_{1} D$ and $S B_{2} D$, and the other along $S B_{3} D$. To estimate the effects of signals along path $S B_{3} D$, we numerically simulate refractions at arbitrary boundaries, at which the locations of source $S$ and detector $D$ are randomly chosen. We find that the $S B_{3} D$-type refraction rarely occurs. Therefore, we expect the artifacts introduced by the signals along $S B_{3} D$ to be insignificant; and, in the following studies, we consider only focusing-type interference.

Analysis of focusing-type interference. For a boundary segment with a size of $2 a$ much larger than the wavelength of interest $\lambda$, we will use a ray model to study the effects of refraction. To have focusing-type interference, the posi- 
tions of the source and detector must satisfy the following equation:

$$
\frac{1}{l_{S B_{1}} / \cos \phi_{1}}+\frac{1}{l_{B_{1} D}(1-\alpha) / \cos \phi_{1}}=\frac{\alpha}{R_{1}}=\frac{1}{f},
$$

where $f$ is the focal length of segment $B_{1} B_{2}$ in Fig. 4 and $f=R_{l} / \alpha ; R_{l}$ is the radius of the segment; and $l_{S B_{1}}$ and $l_{B_{1} D}$ are the lengths of line $S B_{1}$ and $D B_{1}$, respectively. The derivation of (11) can be found in Appendix A. To have a real image, or equivalently to have two rays intersect after passing through boundary segment $B_{1} B_{2},(11)$ requires:

$$
l_{S B_{1}} / \cos \left(\phi_{1}\right)>R_{l} / \alpha .
$$

Next, we derive another requirement due to diffraction for the occurrence of strong amplitude distortion. The smallest beam width after a wavefront passes through a boundary segment with a size of $2 a$ is $l_{B_{1} D} \lambda / a$, where $\lambda$ is the wavelength of the acoustic wavefront. To induce strong focusing, for example, to have a beam width smaller than $a$ at $D$, we need to have:

$$
l_{B_{1} D}<a^{2} / \lambda
$$

The right-hand side of the above inequality is the same as the definition of the near-field length of a plain transducer when $a$ is considered as the radius of the transducer. It is well-known that the amplitude can change rapidly in the near field due to the acoustic interference, but it is much smoother in the far field. Similarly, if the detector is placed within the far field of the concave boundary segment, the amplitude distortion will be less severe in TAT.

Eq. (13) is derived for the case in which a wavefront propagates perpendicularly to the boundary segment. When a wavefront is incident obliquely upon the segment $B_{1} B_{2}$, the effective size of the lens in (13) should be the projection of its geometrical size onto the plane perpendicular to the propagation direction of the incident wave. Then we have:

$$
l_{B_{1} D}<\left(a \cos \phi_{1}\right)^{2} / \lambda \text {. }
$$

By combining (14), (12), and $R_{l}>a$, we obtain the following requirement for inducing strong amplitude distortion after passing through the boundary:

$$
l_{S B_{1}}>\frac{\sqrt{l_{B_{1} D} \lambda}}{\alpha} .
$$

It can be seen from this equation that when $l_{B_{1} D}$ is large enough:

$$
l_{B_{1} D}>\frac{\left(l_{S B_{1}} \alpha\right)^{2}}{\lambda}
$$

the strong amplitude distortion can be minimized. Notice that the required minimum detection distance in (16) increases linearly with the frequency of the wave.
In the derivation of (11), ray theory is utilized. Ray theory is valid under the following conditions [29]:

$$
l_{B_{1} D} \ll 4 a^{2} / \lambda,
$$

and

$$
2 a \gg \lambda \text {. }
$$

Eq. (17) is similar to (13), but the former is stronger; (17) states that the ray model is valid when the wave propagation distance from the heterogeneity is much smaller than $4 a^{2} / \lambda$; beyond that distance, diffraction must be considered. In our analysis of amplitude distortion in TAT, we extend the effective range of the ray model from (17) to (13). This is based on the assumption that the ray model overestimates the wavefront distortions due to ignorance of the diffraction effect. Therefore, if the analysis using ray theory shows that there is only minor amplitude distortion when (16) and (18) are met, the analysis from the exact wave equation should yield the same result.

For a wavelength-scale boundary segment (e.g., $2 a<$ $4 \lambda),(18)$ is violated, and (16) cannot be applied. In this case, strong amplitude distortion can be minimized by placing the detector within the far field of the heterogeneity:

$$
l_{B_{1} D}>4 \lambda
$$

where we have substituted $2 a<4 \lambda$ into (13). Combining (16) and (19), we obtain the minimum detection distance for avoiding strong amplitude distortion induced by different scales of heterogeneities:

$$
l_{B_{1} D}>\max \left[\frac{\left(l_{S B_{1}} \alpha\right)^{2}}{\lambda}, 4 \lambda\right],
$$

where $\max []$ represents computing the maximum. Using the following parameters, $l_{S B_{1}}<10 \mathrm{~cm}$ (the assumed size of the breast parenchyma), and $\alpha=0.07$, in which the mean velocity in the subcutaneous zone $v_{f}$ and the breast parenchyma $v_{p}$ are assumed to be $1437 \mathrm{~m} / \mathrm{s}$ [28] and $1546 \mathrm{~m} / \mathrm{s}$ [30], respectively, we have $l_{B_{1} D}>4.9 \mathrm{~cm}$ for $1.5 \mathrm{MHz}$ ultrasound and $l_{B_{1} D}>1.63 \mathrm{~cm}$ for $0.5 \mathrm{MHz}$ ultrasound. These requirements can be met easily in TAT experiments. For ultrasound waves with a frequency less than $0.5 \mathrm{MHz}$, it is not necessary to apply (20), because ultrasound scattering by soft tissue in this frequency range can be neglected and no severe amplitude distortion is expected.

The above analysis is made for 2-D TAT. This corresponds to the experimental configuration in which a linear, or ring array of transducers with a cylindrical surface is used, and a section image of the breast in the detection plane is desired. However, because of the refraction at the parenchyma wall, the thermoacoustic waves from the objects within the detection plane might deviate out of the plane. On the other hand, the signals collected in the detection plane are transmitted by the objects out of the 
detection plane rather than within it. Consequently, the obtained image is actually a projection of the out-of-plane objects onto the detection plane. To reduce this kind of error, we can use the technique of compressing the breast against the chest wall, which has proven to be effective in reducing wavefront distortions in breast ultrasound imaging. After the compression, the acoustic signals can pass through the interface more or less perpendicularly. However, the ultimate solution to this problem is 3-D TAT. Most of the 2-D results on amplitude distortions (e.g., (14), (16), (20), and the results on phase distortions shown later) can be directly applied to 3-D TAT; (11) also can be applied to analyze a $3-\mathrm{D}$ convex boundary locally by substituting $-R_{l}$ for $R_{l}$. Then, it is straightforward to see that in a 3-D case no two rays can intersect with each other after passing a convex boundary segment.

In summary, our analysis shows that, in RF breast TAT, if the detection is made at a distance to the breast surface required by (20), the amplitude distortion caused by the refraction at the parenchyma wall is not important because of the diffraction effect and the fact that TAT signals are broadband, have low central frequency, and experience only one-way transmission through the parenchyma wall. The effect of intramammary fat lobules will be addressed in Section VI. Therefore, in the following analysis and simulations, we will consider only phase distortion.

\section{B. Phase Distortion Caused by Refraction and Speed Variation}

If the background is acoustically homogeneous, an acoustic ray from source $S$ in Fig. 2 goes along the straight line $S D$ to reach detector $D$. When there is acoustic heterogeneity, an acoustic ray goes along line $S B_{1} D$ because of refraction at the interface. Assume there is no change in the shape of the acoustic pulse caused by acoustic heterogeneity. The TOF from source $S$ to detector $D$ in the acoustically heterogeneous model is:

$$
t_{S B_{1} D}=\int_{S B_{1} D} d l / v_{s}\left(\mathbf{r}^{\prime \prime}\right)
$$

where $v_{s}\left(\mathbf{r}^{\prime \prime}\right)$ is the local acoustic speed, and $\mathbf{r}^{\prime \prime}$ is a point within line $S B_{1} D$. Now, we will show that $t_{S B_{1} D}$ can be approximated to the second order of a small value $\varepsilon=$ $\left(v_{s}\left(\mathbf{r}^{\prime \prime}\right)-v_{s 0}\right) / v_{s 0}$ by $t_{S D}=\int_{S D} d l / v_{s}\left(\mathbf{r}^{\prime \prime}\right)$, where $v_{s 0}$ is the velocity used in the acoustically homogeneous model. According to Fermat's principle, an acoustic ray travels on the fastest path. In other words, $S B_{1} D$ is a local minimum of TOF. Now assume $B_{1}$ is displaced to $B^{\prime}$ by a small distance $q=\left|B B^{\prime}\right|$,

$$
\frac{q}{l_{S D}}=o(\varepsilon)
$$

After expanding $t_{S B^{\prime} D}$ around $t_{S B_{1} D}$ with respect to $q$, we have:

$$
t_{S B^{\prime} D}=t_{S B_{1} D}+\left.q \frac{\partial t_{S B^{\prime} D}}{\partial q}\right|_{q=0}+o\left(\varepsilon^{2}\right) .
$$

Recalling that $S B_{1} D$ is a local minimum, we have $\left.\frac{\partial t_{S B_{1}^{\prime} D}}{\partial q}\right|_{q=0}$. Substituting it into (23) and assuming $l_{B_{2} B_{1}} / l_{S D}=o(\varepsilon)$ due to the weak acoustic heterogeneity in breast tissue, we have:

$$
t_{S D}=\int_{S D} d l / v_{s}\left(\mathbf{r}^{\prime \prime}\right)=t_{S B_{1} D}+o\left(\varepsilon^{2}\right) .
$$

The above result can be understood in the following way. Although the path length of $S B_{1} D$ in Fig. 2 is longer than that of $S D$ and $\left(l_{S B_{1}}+l_{D B_{1}}-l_{S D}\right) / l_{S D}=o(\varepsilon)$, path $S D$ has a longer part within the slow-speed area than path $S B_{1} D$. The combination of the two opposite effects leads to the cancellation of the first-order term of $\varepsilon$ in (24).

Next we will show that the approximation of $t_{S B_{1} D}$ by $t_{S D}$ includes most of the flight-time variation induced by acoustic heterogeneity. The TOF from source $S$ to detector $D$ in an acoustically homogeneous and heterogeneous model is $l_{S D} / v_{s 0}$ and $t_{S B_{1} D}$, respectively. The difference between them is:

$$
\begin{aligned}
\delta t & =\left|t_{S B_{1} D}-l_{S D} / v_{s 0}\right| \\
& =\left|t_{S B_{1} D}-t_{S D}+t_{S D}-l_{S D} / v_{s 0}\right| \\
& \approx\left|o\left(\varepsilon^{2}\right)+t_{S D}-l_{S D} / v_{s 0}\right| \approx o(\varepsilon)
\end{aligned}
$$

where we used (24). Combining $\delta t$ with (24), we have:

$$
\frac{\left|t_{S D}-t_{S B_{1} D}\right|}{\delta t}=o(\varepsilon)
$$

Therefore, the error in the approximation of $t_{S B_{1} D}$ by $t_{S D}$ is not important. At last, it should be pointed out that our analysis of TOF can be applied to both 2-D and 3-D TAT.

\section{Forward Formula in an Acoustically Heterogeneous Model}

In our analysis of TOF, we consider only a single interface. The results can be extended to the case involving several interfaces. In general, the TOF from $\mathbf{r}$ to $\mathbf{r}^{\prime}$ can be expressed as:

$$
t_{f}\left(\mathbf{r}^{\prime}, \mathbf{r}\right)=\int_{L\left(\mathbf{r}^{\prime}, \mathbf{r}\right)} d l / v_{s}\left(\mathbf{r}^{\prime \prime}\right)+o\left(\varepsilon^{2}\right)
$$

where $L\left(\mathbf{r}^{\prime}, \mathbf{r}\right)$ is the straight line from $\mathbf{r}^{\prime}$ to $\mathbf{r}$, and $\mathbf{r}^{\prime \prime}$ lies within the line $L$. Combining (27) and (7), we obtain the forward formula for acoustically heterogeneous TAT.

Our analysis of TOF is in agreement with the results from a more rigid model [31]. It was reported that the variation in travel time caused directly by acoustic speed heterogeneity is a first-order perturbation, and that the effect of the ray bending on the travel times is a secondorder one. For breast tissue, which is weakly acoustically heterogeneous, it is enough to consider the first-order perturbation by computing the integral of the slowness perturbation along straight lines, as shown in (27). 


\section{Implementation AND Modeling OF NUMERICAL Simulations}

\section{A. Numerical Implementation}

It can be seen from $(7)$ that $p_{1}(\mathbf{r}, t)$ can be obtained from $\varphi\left(\mathbf{r}^{\prime}\right)$ after applying two linear operations to it: one is integration over the object space, the other is differentiation over $t$. Therefore, in its discrete form, (7) is a set of linear equations:

$$
\mathbf{M} \varphi=\mathbf{p}
$$

where $\mathbf{M}$ is the matrix representing the product of the two linear operators. The standard techniques of solving a linear equation system can be used. We adopted the TCG method to minimize the object function $\|\mathbf{M} \varphi-\mathbf{p}\|$ in the sense of least square root and no preconditioner is used. In the implementation of TCG, instead of the whole matrix $\mathbf{M}$, a function that gives the multiplication of matrix $\mathbf{M}$ and its adjoin with an arbitrary vector is required. Consequently, the demand on computer memory is reduced greatly, compared with many other techniques that require storing the whole matrix $\mathbf{M}$ in memory. Another advantage of TCG is that an approximate result can be obtained by stopping the iteration before reaching the full convergence. The truncation not only saves computation time but also provides a way of regularization for stabilizing the results. In (28), we use the Savitzky-Golay smoothing method [32], rather than the finite differentiation method to implement the operation of the first-order temporal derivative, as the former yields a much smoother and more accurate result than the latter when data are noisy. We truncated our simulations after 15 iterations, which corresponds to the relative changes in the norms of the results, about $0.7 \%$ for the acoustically homogeneous model and up to $6 \%$ for the acoustically heterogeneous model. In both cases, further iterations yield little visible improvement to image and may induce instability.

In our simulations, we choose the $2-\mathrm{D}$ case rather than the 3 -D case because the computational complexity can be reduced and because it is much easier to interpret and graph a 2-D image. For the 2-D case, the integration in (7) is over a curve instead of a spherical surface:

$$
p_{1}(\mathbf{r}, t)=\frac{\beta I_{0} v_{s 0}}{4 \pi C} \frac{\partial}{\partial t} \oint_{t=t_{f}\left(\mathbf{r}^{\prime}, \mathbf{r}\right)} \frac{\varphi\left(\mathbf{r}^{\prime}\right)}{\left|\mathbf{r}-\mathbf{r}^{\prime}\right|} d \mathbf{r}^{\prime}
$$

where $t_{f}$ is determined by (27). Nevertheless, the conclusions of a $2-\mathrm{D}$ case can be extended to a $3-\mathrm{D}$ one.

\section{B. Model and Parameters in Numerical Simulations}

Fig. 5(a) and (b) illustrate the acoustic and RF absorption models of the breast, respectively. The acoustic model of the breast in our simulations is based on experimental results on the distribution of acoustic speed in the breast [27]-[30]. Acoustic speed in the breast may vary
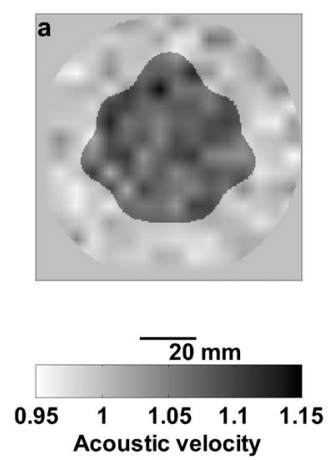
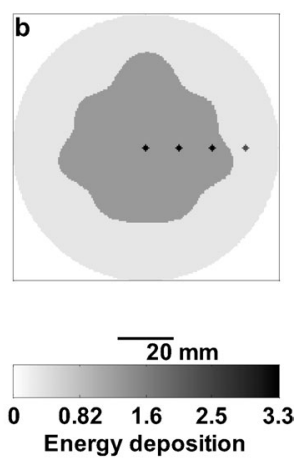

Fig. 5. (a) Distribution of acoustic velocity normalized to $v_{s 0}$ for a breast model. The breast surface is represented by the outer circle; the wall between the breast parenchyma and the subcutaneous fat is represented by the inner irregular boundary. (b) The microwave absorption distribution in our model. The four small spots represent the assumed tumors.

from $1400 \mathrm{~m} / \mathrm{s}$ to $1550 \mathrm{~m} / \mathrm{s}$. Generally, a zone of low velocity $(1400-1450 \mathrm{~m} / \mathrm{s})$ characterizes subcutaneous fat [33]. The speed in normal dense parenchyma is higher, varying from $1500 \mathrm{~m} / \mathrm{s}$ to $1550 \mathrm{~m} / \mathrm{s}$ [30]. In Fig. 5 the outer circles, with a radius of $50 \mathrm{~mm}$, represent the breast surface. The inner irregular boundaries, which are generated by randomly modifying a circle, represent the walls of the breast parenchyma. The size of the parenchyma tissue was changed in different simulations because the ratio of breast parenchyma to subcutaneous fat may change with age. Usually, a young female breast has less fat than an older one does. The mean velocity in the subcutaneous zone $v_{f}$ and the breast parenchyma $v_{p}$ are set to be $1437 \mathrm{~m} / \mathrm{s}$ [27] and $1546 \mathrm{~m} / \mathrm{s}$ [30], respectively. A random component, which is a normal distribution with a mean of zero and a variance of $33 \mathrm{~m} / \mathrm{s}$, is added to the velocity distribution to simulate the velocity fluctuations in the subcutaneous zone [33] and the breast parenchyma [30]. Later, our simulation results show that the random component of velocity will induce little spread in the images due to the cancellation after integration. To ensure that the acoustic speed does not change sharply within each tissue, the random component is smoothed spatially by introducing a correlation length as shown below. The imaged area is divided into patches with side dimensions of a correlation length. The value of the random component at the center of each patch is determined according to the normal distribution mentioned above; then the random component within the patch decreases linearly to zero at the boundary of the patch. We tried different correlation lengths in our simulations, from $12 \mathrm{~mm}$ (about the size of fat lobules in parenchyma tissue) to $3 \mathrm{~mm}$. The image degrades more with increasing correlation length, but the difference is minor. The correlation length was chosen to be about $6 \mathrm{~mm}$ for the reported results. The speed distribution in Fig. 5 was normalized to $1437 \mathrm{~m} / \mathrm{s}$, which is assumed to be the acoustic velocity in the medium surrounding the breast and the mean acoustic speed in the subcutaneous fat. 
The RF absorption model of the breast is shown in Fig. 5(b). The boundary shapes are the same as in Fig. 5(a). The RF absorption coefficients in fat, tumors, and the coupling oil are set to be $0.3,3$, and 0 after being normalized to that in the parenchyma. The tumors, shown in Fig. 5(b) as dark spots, are placed evenly along the horizontal direction to study the dependence of the distortions in the images on the tumor locations. We set the radii of the four tumors to about $1.2 \mathrm{~mm}$ to simulate approximately the point-source spread caused by acoustic heterogeneity.

The parenchyma wall in our simulation is generated as the following equation: $r(\theta)=r_{p}(1+A g(\theta))$, where $r(\theta)$ is the radius of the boundary at angle $\theta, r_{p}$ is the mean radius of the boundary and is used to represent the size of the parenchyma tissue, $A$ is the distortion amplitude, and $g(\theta)$ generates random numbers within $[-1,1]$.

The parameters in our simulations are chosen as follows unless stated otherwise. Noise is added to the generated signals so that the frequency range with signal-to-noise ratio (SNR) larger than unity is from 0 to $1.5 \mathrm{MHz}$, which approximates our experimental results [21]. The radius of the circle of detection is set to be $125 \mathrm{~mm}$ to meet (16); the angle range of detection is $2 \pi$ with 400 steps. An insufficient number of scanning steps can cause radial aliases in the reconstructed image [13]. Thermoacoustic signals are sampled for $108 \mu$ s at a sampling rate of about $14 \mathrm{MHz}$, which is sufficient to meet the Nyquist criteria. The $100 \mathrm{~mm}$ by $100 \mathrm{~mm}$ imaging field is mapped with a 256 by 256 mesh. In our simulations, the thermoacoustic signals are generated in an acoustically inhomogeneous model, and the reconstruction is implemented for two cases - with and without the consideration of acoustic heterogeneity.

\section{Numerical Results}

We first study the effect of acoustic heterogeneity on imaging when acoustic heterogeneity is considered in the forward problem but not in the reconstruction. In the reconstruction, $v_{s}(\mathbf{r})$ in $(27)$ is set to be $v_{s 0}$. Then we show how to improve image resolution after considering acoustic heterogeneity in the reconstructions. And, the effects of measurement errors in $v_{f}, v_{p}$ and $\Sigma$ on the improvement are investigated.

\section{A. Reconstruction Without Considering Heterogeneity}

Fig. 6(a)-(d) shows the results when acoustic heterogeneity is not considered in the reconstructions. In the four simulations, the mean radii of the parenchyma wall $r_{p}$ are set to be $0.8,0.6,0.4$, and 0.2 of the breast radius. The wall is distorted randomly in the simulations, and the distortion amplitude is 0.1 . We measure the point-spread width (PSW), which is the width of the image of a point source along a specific direction minus its real size, $2.4 \mathrm{~mm}$, and the boundary spread width (BSW), which is the width of the blurred parenchyma wall $\Sigma$ in an image. It is clear from
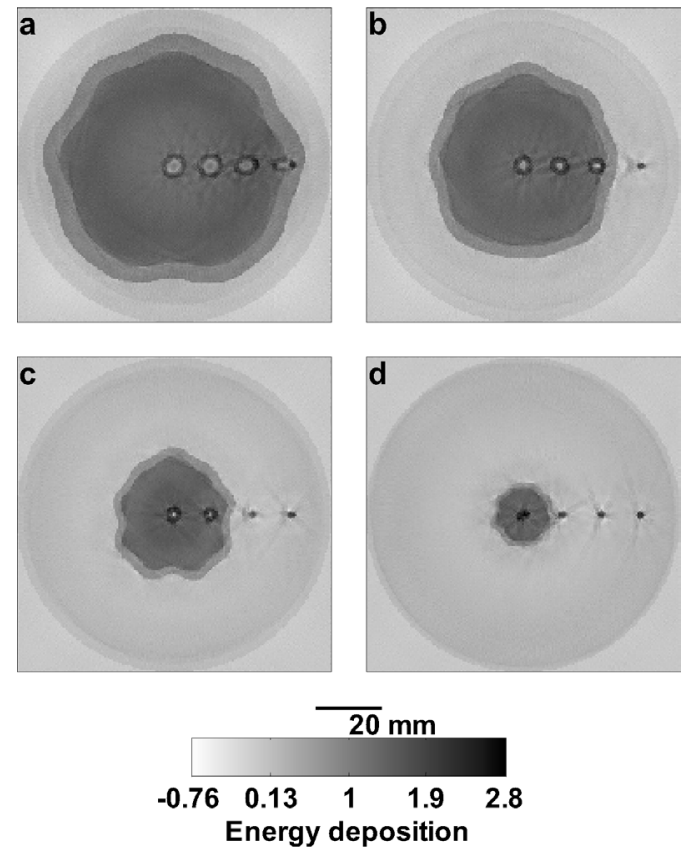

Fig. 6. Images when acoustic heterogeneity is not considered in the reconstructions. The mean radii of the parenchyma wall are set to be (a) 0.8 , (b) 0.6 , (c) 0.4 , and (d) 0.2 of the breast radius, respectively. The point-spread width and the boundary-spread width increase linearly with the size of the parenchyma tissue. Note that the spread of points outside the parenchyma tissue are much smaller than the spread inside.

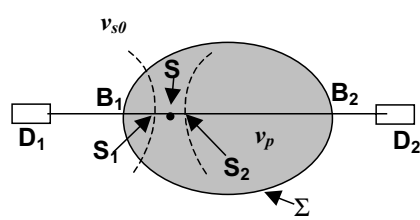

Fig. 7. Diagram for deriving (30), which estimates the spread of a point source $S$ along line $D_{1} D_{2}$ due to TOF error. $S_{1}$ is the intersection of $D_{1} D_{2}$ with the backprojection arch of the signal transmitted by source $S$ and detected by detector $D_{1} ; S_{2}$ is the corresponding one at $\mathrm{D}_{2}$.

Fig. 6 that PSW and BSW increase with the radius of the parenchyma wall. It is proved in Appendix B that the two widths can be estimated by the following equation:

$$
w=l_{p} \alpha,
$$

where $l_{p}$ is $2 r_{p}$ in the case of BSW; in the case of PSW, $l_{p}$ is the length of a ray within the parenchyma tissue along a specific direction (for example the length of $B_{1} B_{2}$ in Fig. 7). The PSW is anisotropic because $l_{p}$ depends on direction. This anisotropy of PSW can be verified by the observation that the three tumors within the parenchyma tissue in Fig. 6(a) and (b) have the same spread along the horizontal direction, and their spreads along the vertical direction decrease when the tumors are located away from the center. 


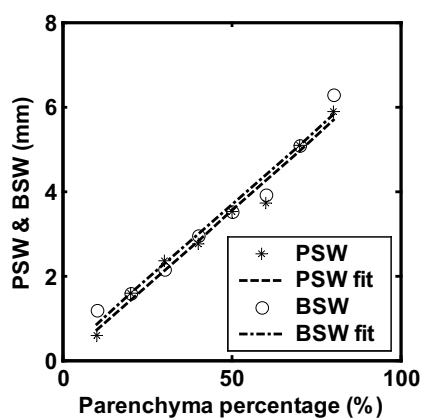

Fig. 8. Quantitative results of the point-spread width and boundaryspread width along the horizontal direction in eight simulations in which the mean radius of the parenchyma wall changes from 0.1 to 0.8 of the breast radius using a step of 0.1 . The corresponding linear fittings of PSW (dashed) and BSW (dash-dotted) are in good agreement with the proposed formula (30).

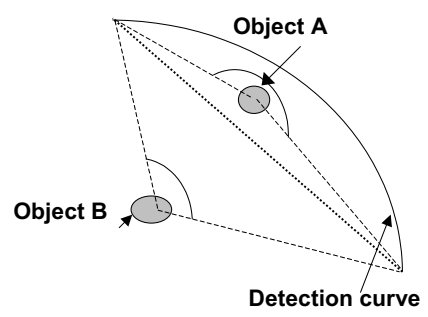

Fig. 9. Diagram showing that in TAT a $\pi$ or wider view can provide complete data for reconstruction. A view means the angle subtended by the detection curve when observed from the to-be-imaged object. Object $A$ has a view larger than $\pi$, and object $B$ has a view less than $\pi$.

Fig. 8 shows the quantitative results (with an error of $\pm 0.8 \mathrm{~mm}$ ) of the PSW and BSW along the horizontal direction in eight simulations in which the radius changes from 0.1 to 0.8 of the breast radius with a step of 0.1 . The corresponding linear fitting results for the PSW and BSW are shown as dashed and dash-dotted lines, respectively. The slopes of the two lines are 0.071 and 0.0705 , respectively, both of which are close to the estimated rate of 0.07 derived from (30) after substituting the parameters used in our simulations, the radius of the breast $r_{b}=50 \mathrm{~mm}$ and $\alpha=0.07$.

Another interesting point in Fig. 6 is that the PSW of the objects outside the parenchyma tissue are affected little by acoustic heterogeneity. Only minor artifacts are observed near them. This is because in TAT a $\pi$ or wider view can provide complete data for reconstruction [34]. Here, a view means the angle subtended by the detection curve when observed from the to-be-imaged object. For example, object $A$ in Fig. 9 has a view larger than $\pi$, and object B's is less than $\pi$. If an object is outside the parenchyma tissue, it has at least a $\pi$-view detection range in which the medium between the object and the detectors is acoustically homogeneous. Therefore, a perfect image can be reconstructed from this part of the data. On the other hand, the image reconstructed from the part of signals that experience the heterogeneous medium is weak
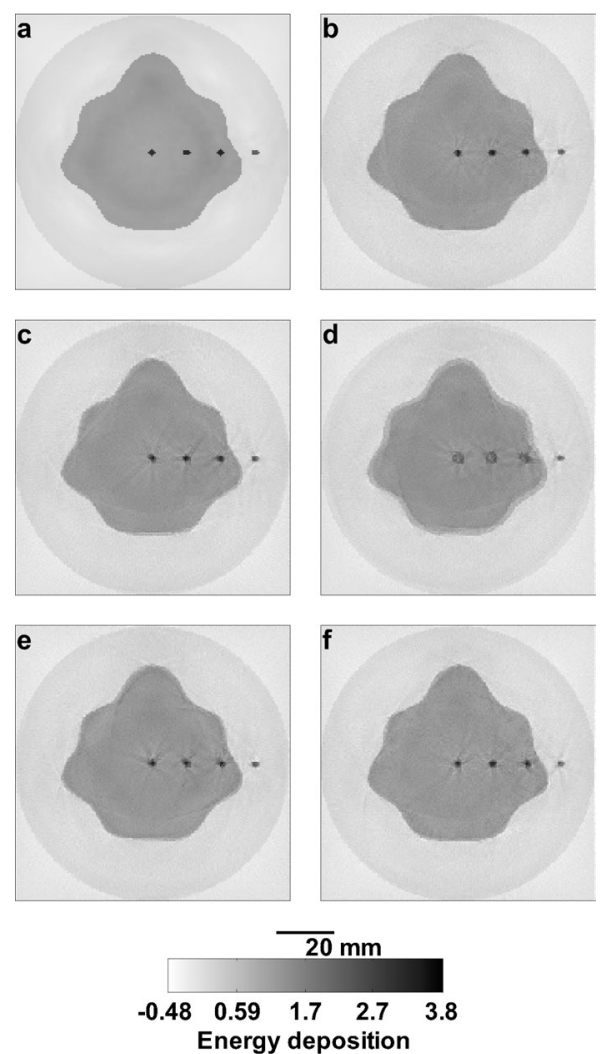

Fig. 10. (a) Compensation for the degradation in images when complete acoustic heterogeneity information is included in the reconstructions. (b) Only exact $v_{p}, v_{f}$, and $\Sigma$ are included to show the insensitivity of improvement to a random component of the acousticvelocity distribution. (c) and (d) Images in which there are (c) $1 \%$ and (d) $3 \%$ errors in $v_{p}$, respectively. (e) Images in which $\Sigma$ is scaled down by $10 \%$. (f) Images in which $20 \%$ random error is introduced in $\Sigma$. These results show the stability of the improvement to the errors in $v_{p}, v_{f}$, and $\Sigma$.

in amplitude because the flight-time errors compromise the build-up strength of the signals.

In addition to blurring of images, acoustic heterogeneity increases the background noise level and decreases the values of reconstructed tumors, which consequently reduces the contrast of tumors in the images and the detectability of small tumors. A comprehensive quantitative study of this issue will depend on the SNR of the hardware of the imaging system, the parameters of the imaging system and reconstruction algorithms, and the contrast of the to-beimaged objects. Meaningful conclusions should be made based on relevant experimental data which we leave for future study.

\section{B. Reconstruction with the Consideration of Heterogeneity}

The exact distribution of acoustic velocity is included in the model in Fig. 10(a). Although the result is good, it is not practical, because it is not feasible to obtain the exact distribution of velocity in the breast by current technology. A much more practical situation is when the mean velocities $v_{f}, v_{p}$, and boundary profile $\Sigma$ are approximately known and the velocity fluctuation within each area is un- 

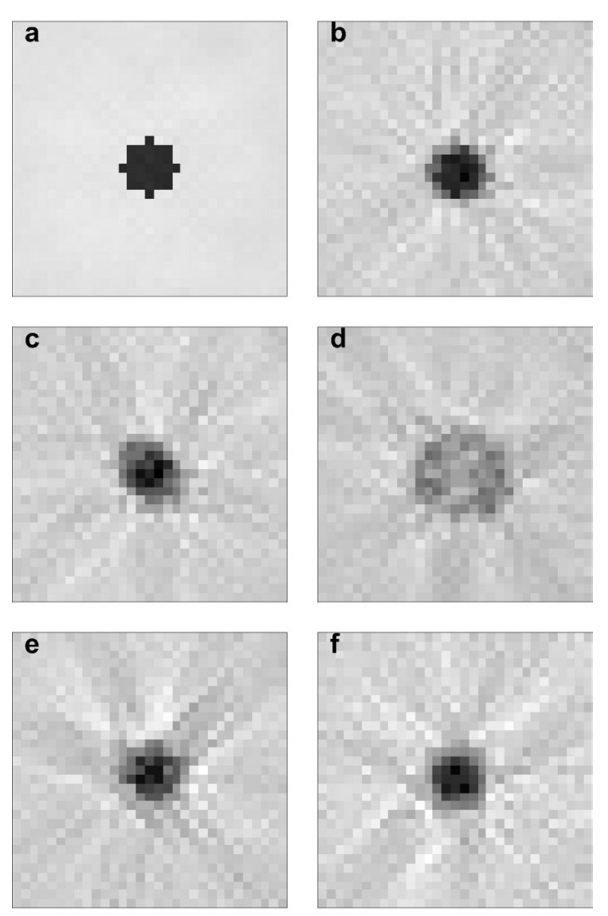

$2 \mathrm{~mm}$

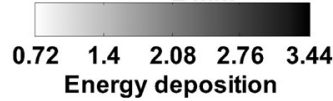

Fig. 11. (a)-(f) Close-up images around the central tumor in Fig. 10. Compensation for the degradation in images when complete acoustic heterogeneity information is included in the reconstructions. (b) Only exact $v_{p}, v_{f}$, and $\Sigma$ are included to show the insensitivity of improvement to a random component of the acoustic-velocity distribution. (c) and (d) Images in which there are (c) $1 \%$ and (d) $3 \%$ errors in $v_{p}$, respectively. (e) Image in which $\Sigma$ is scaled down by $10 \%$. (f) Image in which $20 \%$ random error is introduced in $\Sigma$.

known. Different approaches to obtain $v_{f}, v_{p}$, and boundary profile $\Sigma$ will be explored in Section VI. Here, we will show the effectiveness of our compensation method. Figs. 10(b)-(f) show the images reconstructed from the same data as in Fig. 10(a), but the reconstruction algorithm used only $v_{f}, v_{p}$, and $\Sigma$ to study the effects of the measurement errors in $v_{f}, v_{p}$, and $\Sigma$ on the improvement. In Figs. 10(b)-(f), the random component of the acousticvelocity distribution is ignored. In addition, $v_{p}$ is decreased by $1 \%$ and $3 \%$ in Figs. 10 (c) and (d), respectively; $\Sigma$ is scaled down by $10 \%$ in Fig. $10(\mathrm{e})$; and a $20 \%$ random error is introduced to $\Sigma$ in Fig. 10(f). Figs. 11(a)-(f) are the corresponding close-up images around the central tumor in Fig. 10. The $r_{p}$ in these simulations is 0.6 of the breast radius, and the distortion amplitude of the parenchyma wall is 0.2 .

1. Effect of Errors in Velocities: There is little difference between the resolution of the reconstructed images when we consider [Fig. 11(a)] and do not consider [Fig. 11(b)] the random component of velocity distribution, although the artifacts in the background in Fig. 11(b) are a little stronger than those in Fig. 11(a). The good resolution, after ignoring the random component of the acoustic-velocity distribution in Fig. 11(b), can be explained by modifying (30) to:

$$
w=\int_{B_{1} B_{2}} \alpha\left(\mathbf{r}^{\prime \prime}\right) d l_{p},
$$

where $\alpha\left(\mathbf{r}^{\prime \prime}\right)=1-v_{s 0} / v_{p}\left(\mathbf{r}^{\prime \prime}\right)$ and is spatially dependent; the integration is over the line $B_{1} B_{2}$ in Fig. 7. It can be found that the contributions of the random component of velocity are canceled in some degree after the integration over an acoustic ray.

Comparing Figs. 11(c) and (d) with Fig. 11(b), it can be noticed that a $1 \%$ error in $v_{p}$ does not degrade the imaging quality much, but a $3 \%$ error in $v_{p}$ greatly deteriorates the imaging resolution and contrast. This is because in our model the difference between $v_{f}$ and $v_{p}$ is about $7 \%$ of their speeds, and a $3 \%$ error in $v_{p}$ actually accounts for $42 \%$ of the difference between $v_{f}$ and $v_{p}$. Therefore, we conclude that an accuracy of $1 \%$ in the determination of $v_{p}$ is sufficient for significant improvement in imaging resolution.

2. Effects of Errors in Determining $\Sigma$ : In the model in Fig. 11(e), the boundary $\Sigma$ is scaled down by $10 \%$. In Fig. 11(f), a random component is added to the real boundary, which is implemented by multiplying the real radii of a boundary with uniform random numbers within $[0.8,1.2]$. After comparing Figs. 11(e) and (f) with other components of Fig. 11, it is found that compensation is less sensitive to error in determining $\Sigma$ as $v_{p}$. This is because a $10 \%$ error, which is about $6 \mathrm{~mm}$ in the diameter of the parenchyma wall, adds an error of at most $0.42 \mathrm{~mm}$ to the PSW and BSW according to (30).

\section{Discussion}

\section{A. Effect of Small Fat Lobules}

In breast UT, centimeter-scale fat lobules in the parenchyma tissue also can cause significant distortion. In breast TAT, the amplitude distortion due to centimeterscale fat lobules is estimated to be insignificant because of the diffraction effect, as discussed in Section III-A,2. For example, substituting $a=1 \mathrm{~cm}, \lambda=1.5 \mathrm{~mm}$ in (13), we obtain a near-field length of $6.7 \mathrm{~cm}$. Therefore, no strong amplitude distortion is expected when detectors are placed farther than $6.7 \mathrm{~cm}$ from the lobule. In addition, Figs. 6 (b)-(d) show that the images of point sources outside an acoustic heterogeneity are affected little by the acoustic heterogeneity due to the completeness of the $\pi$-view detection in TAT. This explanation also can be applied to the distortion caused by fat lobules. When a fat lobule on one side of an acoustic source causes severe distortion, the signals that are spared from severe distortion in other directions still can produce good images. 


\section{B. Determine $v_{f}$ and $v_{p}$ in Experiments}

Our simulation results in Fig. 10(c) and Fig. 11(c) show that a $1 \%$ error in $v_{f}$ and $v_{p}$ will lead to minor blurring but that we still have enough definition to determine the configuration and location of the imaged objects. To determine $v_{f}$ and $v_{p}$ within $1 \%$ accuracy, we can try different speeds around the averages, which are $1437 \mathrm{~m} / \mathrm{s}$ and $1546 \mathrm{~m} / \mathrm{s}$ for fat and breast tissue, respectively, with a step size of $1 \%$ velocity. Optimum speeds can be determined by choosing the reconstructed image with the sharpest parenchyma wall, because errors in $v_{f}$ and $v_{p}$ will cause the spread of this boundary. Because the variations of $v_{f}$ and $v_{p}$ between individuals are about $2 \%$ and $4 \%$, respectively, only 15 trials are needed to scan all the combinations. Furthermore, the backprojection method [12] can be used in each trial because the boundary of the reconstructed image can be recovered well with this method [34], [35]. Therefore, the additional computation cost in the trials is estimated to be only double the total computation complexity.

\section{Determine $\Sigma$ in Experiments}

There are two ways to obtain $\Sigma$. The first method uses only TAT signals. It takes advantage of the fact that fat and parenchyma have both acoustic and microwave contrasts. A TAT image is first reconstructed with an acoustically homogeneous model. Then an approximate $\Sigma$ can be extracted from the image and plugged into an acoustically heterogeneous reconstruction model to obtain a more accurate TAT image. As shown in Fig. 6, the boundary spread of the parenchyma wall in TAT images is at most $7 \%$ of its real size (if $\alpha=0.07$ ) when an acoustically homogeneous reconstruction model is used. Our studies of the effects on the reconstruction of the errors in the boundary profile, shown in Fig. 10(e) and Fig. 11(e), reveal that this level of error has little effect on the images reconstructed from a heterogeneous model. We intend to implement this method in our future work.

The second method for determining $\Sigma$ is the coregistration of ultrasound B-scan imaging and TAT. In principle, this can be accomplished in the same set-up. The TAT data is acquired, then the transducers work in pulse-echo mode to determine an approximate $\Sigma$. This boundary information can be included in the reconstruction algorithm of TAT.

\section{Differences Between TAT and UT}

The studies we presented in Section III show that there should be no severe amplitude distortion in breast TAT, but severe amplitude distortion caused by refraction has been observed in both narrowband and broadband breast UT [15]. The difference between the effects of acoustic heterogeneity on TAT and UT can be explained by the different central frequencies. In UT, the central frequency is above $3 \mathrm{MHz}$, and in TAT the central frequency is below $1 \mathrm{MHz}$. The higher frequency in UT results in stronger wavefront distortion due to the following reasons. First, the scattering effect increases rapidly with frequency; second, the minimum detection distance for avoiding strong amplitude distortion caused by an acoustic lens, which can be a boundary segment or a small inclusion, extends farther with increasing frequency. Substituting the following parameters for UT, $l_{S B_{1}}<10 \mathrm{~cm}, \lambda=0.5 \mathrm{~mm}$, and $\alpha=0.07$ into (20), we have $l_{B_{1} D}>9.8 \mathrm{~cm}$. We notice that the transducer or array was placed closer than the required distance to the breast [15], [16]. Therefore, it is not surprising to observe the strong interference effect in UT.

Another important difference between TAT and UT is that there is no speckle in our TAT images [11]. Speckle is an important factor limiting the quality of pure ultrasonic imaging. In our technology, the detected signals are primary acoustic waves rather than reflective or scattered waves as in UT. Furthermore, the temporal frequency of the acoustic signals lies in a range from 0 to $1.5 \mathrm{MHz}$, which is only weakly scattered in the tissues. However, the issue of image speckle in more realistic medical imaging applications is a topic for future consideration.

\section{E. Miscellaneous}

Our analysis and numerical simulations have shown that breast TAT images can survive acoustic heterogeneity. The ultimate test, however, will come from clinical experiments on the breast in which the motion artifacts due to breathing and cardiac movement may introduce blurring. Such blurring of images is estimated to be on the order of the movement amplitude. To correct the blurring, we can monitor the breast motion, for example, placing a microwave absorber on the breast surface as a marker. Then the data on the breast motion can be used in the reconstructions to shift the detectors' positions and, consequently, compensate for the breast's displacement.

\section{Conclusions}

The effects of acoustic heterogeneity on TAT in the breast are studied. Our analysis shows that the amplitude distortion in the breast TAT is minor. There is no multipath interference in the breast TAT with a convex parenchyma wall, and the amplitude distortion also is not severe for concave boundary, because the TAT signals are broadband, have low central frequency, and experience only one-way transmission through the parenchyma wall. Therefore we consider only phase distortion in our numerical studies. The numerical results on the spread of point sources and boundaries caused by the phase distortion are in good agreement with the predictions of the proposed formula. It is shown that phase distortion can be compensated for when complete or partial information on the distribution of acoustic velocity in the breast is included in the reconstruction. It is found that improvement in the re- 
sults is more sensitive to measurement error in $v_{f}, v_{p}$ than $\Sigma$. Based on this sensitivity study, an approach to implement our compensation method using only TAT data is proposed. The differences between breast TAT and breast ultrasound imaging in relation to the effects of acoustic heterogeneity and speckles are accounted for by differences in their central frequency of ultrasound and detection configuration.

\section{Appendix A}

\section{DERIVATION OF (11)}

Assume that the concave boundary can be approximated by an arch with a radius $R_{l}>a$, where $a$ is half the size of the boundary segment. Two rays are refracted at points $B_{1}$ and $B_{2}$ in Fig. 4 , where $B_{2}$ has a small displacement from $B_{1}$ along the boundary. According to the refraction law, we have:

$$
\begin{aligned}
\sin \theta_{1} & =(1-\alpha) \sin \phi_{1}, \\
\cos \theta_{1} d \theta_{1} & =(1-\alpha) \cos \phi_{1} d \phi_{1},
\end{aligned}
$$

where $d \phi_{1}$ is the difference between the incidence angles of the two rays and $d \theta_{1}$ is the transmission one. They can be expressed as:

$$
\begin{aligned}
d \theta_{1} & =\left(1-\frac{R_{l} \cos \theta_{1}}{l_{B_{1}} D}\right) d \theta \\
d \phi_{1} & =\left(\frac{R_{l} \cos \phi_{1}}{l_{S B_{1}}}+1\right) d \theta
\end{aligned}
$$

where $l_{S B_{1}}$ and $l_{B_{1} D}$ are the distances from the boundary point $B_{1}$ to source $S$ and detector $D$, respectively, and $d \theta=l_{B_{1} B_{2}} / R_{l}$. Combining the above equations, we have the imaging formula for the boundary segment:

$$
\frac{\cos ^{2}}{l_{S B_{1}}}+\frac{\cos ^{2} \theta_{1}}{l_{D B_{1}}(1-\alpha)}=\frac{\cos \theta_{1} /(1-\alpha)-\cos \phi_{1}}{R_{l}} .
$$

Because in our breast model $\alpha \approx 0.1$ is small, the above equation can be further simplified to (11) after using $\theta_{1} \approx$ $\phi_{1}$.

\section{Appendix B}

\section{DERIVATION OF (30)}

The first iteration in TCG is equivalent to the backprojection method [34]. In backprojection for an acoustically homogeneous TAT, $p(\mathbf{r}, t)$, the signal detected at $\mathbf{r}$ and time $t$ is projected back to a sphere with a radius of $t v_{s 0}$ and a center at $\mathbf{r}$. It is shown that the boundaries of objects can be reconstructed correctly with the backprojection method [35]. Let us consider a model illustrated in Fig. 7 to estimate the spread of source $S$ along line $D_{1} D_{2}$, where $D_{1}$ and $D_{2}$ are two detectors, $S_{1}$ is the intersection of $D_{1} D_{2}$ with the backprojection arch of the signal transmitted by source $S$ and detected by detector $D_{1}, S_{2}$ is the corresponding one at $D_{2}$, and $\Sigma$ represents the parenchyma wall. If there is no error in computing TOFs, $S_{1}, S_{2}$, and $S$ will be one point; therefore, a point image of source $S$ can be recovered. In an acoustically heterogeneous model, however, the flight-time errors caused by the approximation of $v_{p}$ by $v_{s 0}$ in the reconstruction result in the splitting of $S_{1}$ and $S_{2}$ from $S$, where $l_{S_{1} S}$ and $l_{S_{2} S}$ can be estimated by the multiplication of the flighttime errors with $v_{s 0}, l_{S_{1} S}=l_{B_{1} S}\left(1-v_{s 0} / v_{p}\right)=\alpha l_{B_{1} S}$ and $l_{S_{2} S}=l_{B_{2} S}\left(l-v_{s 0} / v_{p}\right)=\alpha l_{B_{2} S}$. Combining them, we have (30) for the spread width of source $S$ along line $D_{1} D_{2}$. Similar analysis can be applied to estimating BSW as well.

\section{ACKNowledgments}

We would like to thank Dr. Q. Zhu for many useful discussions. We also are indebted to the anonymous reviewers, whose constructive comments lead us to a more comprehensive investigation of the problem.

\section{REFERENCES}

[1] W. Joines, R. Jirtle, M. Rafal, and D. Schaeffer, "Microwave power absorption differences between normal and malignant tissue," Radiation Oncology-Biology-Physics, vol. 6, pp. 681-687, 1980.

[2] S. Chaudhary, R. Mishra, A. Swarup, and J. Thomas, "Dielectric properties of normal human breast tissues at radiowave and microwave frequencies," Indian J. Biochem. Biophys., vol. 21, pp. 76-79, 1984.

[3] W. Joines, Y. Zhang, C. Li, and R. Jirtle, "The measured electrical properties of normal and malignant human tissues from 50-900 MHz," Med. Phys., vol. 21, pp. 547-550, 1994.

[4] L. E. Larsen and J. H. Jacobi, Eds. Medical Applications of Microwave Imaging. Piscataway, NJ: IEEE Press, 1986.

[5] S. Caorsi, A. Frattoni, G. L. Gragnani, E. Nortino, and M. Pastorino, "Numerical algorithm for dielectric-permittivity microwave imaging of inhomogeneous biological bodies," Med. Biol. Eng. Comput., vol. NS-29, pp. 37-44, 1991.

[6] M. S. Hawley, A. Broquetas, L. Jofre, J. C. Bolomey, and G. Gaboriaud, "Microwave imaging of tissue blood content changes," J. Biomed. Eng., vol. 13, pp. 197-202, 1991.

[7] P. M. Meaney, K. D. Paulsen, and J. T. Chang, "Nearfield microwave imaging of biologically-based materials using a monopole transceiver system," IEEE Trans. Microwave Theory Tech., vol. 46, pp. 31-45, 1998.

[8] R. A. Kruger, P. Liu, Y. R. Fang, and C. R. Appledorn, "Photoacoustic ultrasound (PAUS) - reconstruction tomography," Med. Phys., vol. 22, pp. 1605-1609, 1995.

[9] C. G. A. Hoelen, F. F. M. Demul, R. Pongers, and A. Dekker, "Three-dimensional photoacoustic imaging of blood vessels in tissue," Op. Lett., vol. 23, pp. 648-650, 1998.

[10] G. Ku and L.-H. V. Wang, "Scanning thermoacoustic tomography in biological tissue," Med. Phys., vol. 27, pp. 1195-1202, 2000.

[11] Y. Xu, D. Feng, and L.-H. V. Wang, "Exact frequency-domain reconstruction for thermoacoustic tomography: I. Planar geometry," IEEE Trans. Med. Imag., vol. 21, pp. 823-828, 2002.

[12] M. Xu and L.-H. V. Wang, "Time-domain reconstruction for thermoacoustic tomography in a spherical geometry," IEEE Trans. Med. Imag., vol. 21, pp. 814-822, 2002.

[13] Y. Xu, M. Xu, and L.-H. V. Wang, "Exact frequency-domain reconstruction for thermoacoustic tomography: II. Cylindrical geometry," IEEE Trans. Med. Imag., vol. 21, pp. 829-833, 2002. 
[14] M. Moshfeghi and R. C. Waag, "In vivo and in vitro ultrasound beam distortion measurements of a large aperture and a conventional aperture focused transducer," Ultrasound Med. Biol., vol. 5, pp. 415-428, 1988.

[15] Q. Zhu and B. D. Steinberg, "Large-transducer measurements of wavefront distortion in the female breast," Ultrason. Imag., vol. 14, pp. 276-299, 1992.

[16] C. W. Manry and S. L. Broschat, "FDTD simulations for ultrasound propagation in a 2-D breast model," Ultrason. Imag., vol. 18, pp. 25-34, 1996.

[17] P. D. Freiburger, D. C. Sullivan, B. H. Leblanc, S. W. Smith, and G. E. Trahey, "Two dimensional ultrasonic beam distortion in the breast: In vivo measurements and effects," Ultrason. Imag., vol. 14, pp. 398-414, 1992.

[18] S. W. Flax and M. O'Donnell, "Phase aberration correction using signals from point reflectors and diffuse scatterers: Basic principles," IEEE Trans. Ultrason., Ferroelect., Freq. Contr., vol. 35, pp. 758-767, 1988.

[19] G. E. Trahey, D. Zhao, J. A. Miglin, and S. W. Smith, "Experimental results with a real-time adaptive ultrasonic imaging system for viewing through distorting media," IEEE Trans. Ultrason., Ferroelect., Freq. Contr., vol. 37, pp. 418-427, 1990.

$[20]$ Q. Zhu and B. D. Steinberg, "Deaberration of incoherent wavefront distortion: An approach toward inverse filtering," IEEE Trans. Ultrason., Ferroelect., Freq. Contr., vol. 44, pp. 575-589, 1997.

[21] Y. Xu and L.-H. V. Wang, "Signal processing in scanning thermoacoustic tomography in biological tissues," Med. Phys., vol. 28, pp. 1519-1524, 2001.

[22] P. C. Hansen, Rank-Deficient and Discrete Ill-Posed Problems. Philadelphia, PA: SIAM Press, 1998.

[23] A. V. Oppenheim and J. S. Lim, "The importance of phase in signals," Proc. IEEE, pp. 529-541, 1981.

[24] B. D. Steinberg, "A theory of the effect of hard limiting and other distortions upon the quality of microwave images," IEEE Trans. Acoust. Speech Signal Processing, vol. ASSP-35, pp. 1462-1472, 1987.

[25] V. E. Gusev and A. A. Karabutov, Laser Optoacoustics. New York: American Institute of Physics, 1993.

[26] A. R. Selfridge, "Approximate properties in isotropic materials," IEEE Trans. Sonics Ultrason., vol. SU-32, pp. 381-394, 1985.

[27] F. S. Foster, M. Strban, and G. Austin, "The ultrasound microscope: Initial studies of the breast tissue," Ultrason. Imag., vol. 6, pp. 243-261, 1984.

[28] M. Born and E. Wolf, Principles of Optics. New York: Cambridge University Press, 1997.

[29] L. A. Chernov, Wave Propagation in a Random Medium. New York: Dover, 1960.

[30] G. Kossoff, E. K. Fry, and J. Jellins, "Average velocity of ultrasound in the human female breast," J. Acoust. Soc. Amer., vol. 53, pp. 1730-1736, 1973.

[31] R. Sneider and D. F. Aldridge, "Perturbation theory for travel times," J. Acoust. Soc. Amer., vol. 98, pp. 1565-1569, 1995.

[32] H. P. William, A. T. Saul, T. V. William, and P. F. Brian, Numerical Recipes in C. Cambridge: Cambridge Univ. Press, 1992.
[33] J. F. Greenleaf and R. C. Bahn, "Clinical imaging with transmissive ultrasonic computerized tomography," IEEE Trans. Biomed. Eng., vol. BME-28, no. 2, pp. 177-185, 1981.

[34] Y. Xu and L.-H. V. Wang, "Limited-view thermoacoustic tomography and reconstruction by truncated-conjugate gradient," IEEE Trans. Med. Imag., submitted for publication.

[35] A. K. Louis and E. T. Quinto, "Local tomographic methods in sonar," in Surveys on Solution Methods for Inverse Problems. Vienna: Springer, 2000, pp. 147-154.

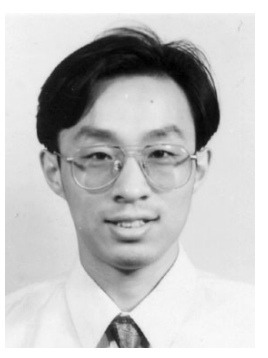

Yuan $\mathbf{X u}$ was born in Guangshui, China in 1971, received his Ph.D. degree in physics from the Institute of Physics, Chinese Academy of Sciences, Beijing, China, in 1999. He is studying for a Ph.D. degree in Biomedical Engineering at Texas A\&M University, College Station, Texas.

His research interest is thermoacoustic tomography.

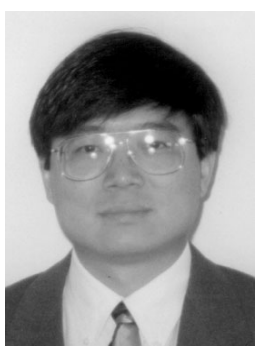

Lihong V. Wang (M'96-SM'00) was born in Guangshui, China on March 8, 1964, received his Ph.D. degree in electrical engineering from Rice University, Houston, TX, in 1992.

Dr. Wang worked for Dr. Steven Jacques initially as a postdoctoral research associate and was shortly promoted to assistant professor at the University of Texas M. D. Anderson Cancer Center, Houston, TX, a topranked cancer institution. He was promoted to professor in biomedical and electrical engition, TX, in 2002. neering, Texas A\&M University, College Sta-

$\mathrm{He}$ is a senior member of the Institute of Electrical and Electronics Engineers (IEEE), and a member of the American Physical Society (APS), Society of Photo-Optical Instrumention Engineers (SPIE), Optical Society of America (OSA), and Engineering in Medicine and Biology Society (EMBS). He received the National Institutes of Health (NIH) First award, National Science Foundation (NSF) Career award, Johnson \& Johnson Outstanding Young Scientist award, Texas A\&M Texas Engineering Experiment Station (TEES) Select Young Faculty award, Texas A\&M TEES Faculty Fellow award (twice), Texas A\&M Ernest A. Baetz Faculty Fellow award, and Texas A\&M University Faculty Fellow award. He is listed in Who's Who in Science and Engineering. He served as a grant reviewer for NIH, NSF, the Navy, and other funding agencies. He is an associate editor for the Annals of Biomedical Engineering, Journal of Biomedical Optics, and Applied Optics. 